\title{
Visual dominance and attention in space
}

\author{
FRANCIS B. COLAVITA \\ University of Pittsburgh, Pittsburgh, Pennsylvania 15260
}

\begin{abstract}
When human subjects are presented a light and a tone simultaneously, each of which serves as a cue in a reaction time task, responding is predominantly to the light. This phenomenon has been referred to as visual dominance. The present experiment attempted to test the hypothesis that when subjects must divide their attention between different objects in space, the visual channel is favored over the auditory channel. Rather than the "free-field" method used in our earlier work, our procedure involved the use of earphones to deliver the auditory stimulus. The results provided a further indication of the robustness of the visual dominance effect. While there was a slight reduction in the magnitude of the effect, as compared with the "free-field" situation, responding was still predominantly to the visual stimulus.
\end{abstract}

Previous studies conducted in our laboratory over the past several years have demonstrated that when human subjects are presented a light and a tone simultaneously, each of which individually serves as a cue in a reaction time (RT) task, subjects respond predominantly to the light. This visual dominance effect is seen to persist when the subjective intensity of the tone is double that of the light (Colavita, 1974), when subjects are given verbal instructions to respond to the tone rather than to the light (Colavita, 1974), when the tone source rather than the light source is used as a visual fixation point (Colavita, Tomko, \& Weisberg, 1976), and when subjects are required to respond to stimulus offset rather than stimulus onset (Colavita \& Weisberg, 1979).

The purpose of the present experiment was to explore a further aspect of this visual dominance effect. More specifically, we wished to test the idea that the subject has a finite amount of attention to direct to objects in space and that, for some reason, the visual channel receives a disproportionate amount of this quantity, to the detriment of attention to auditory events in space.

We felt that one way to test this idea would be to deliver the auditory stimulus directly to the subject's ears via earphones rather than "free field," as had been the method of tone presentation used in our earlier experiments. The use of earphones should free the subject from the necessity of attending to the location of the tone in space and, possibly, bring about some change in the visual dominance effect.

Simple tonal RT is generally reported to be faster than simple light RT. However, in the case of simultaneous light-tone presentation (what we call conflict trials), the subject's responding is dominated by the visual stimulus. Possibly, freeing the subject from the task of dividing his or her attention between two points in space (i.e., the sound source and the light source) through the use of earphones will reduce or abolish the visual dominance effect due to the greater speed of simple tonal RT over simple visual RT. On the other hand, the use of earphones might permit the subject to direct all of his or her attention in space to the light source, thereby enhancing the visual dominance effect.

\section{METHOD}

\section{Subjects}

Subjects were 10 undergraduate psychology students from the University of Pittsburgh. The group was composed of five males and five females. All subjects were right-handed.

\section{Procedure}

Each subject matched an auditory and a visual stimulus for subjective intensity by adjusting a 6 -W light source until it was as bright as a 4,000-Hz $80-\mathrm{dB}$ SPL tone was loud. Then, the matched stimuli served as cues in an RT task. The tone was presented dichotically over Telephonics TDH-49 earphones. The light source was a $6-\mathrm{W}$ incandescent light bulb located in a stimulus panel $45 \mathrm{~cm}$ in front of the subject. The subject's hands were positioned over two telegraph keys, one of which was designated as the tone key and the other, as the light key. These key designations were reversed for half of the subjects. Then, each subject was told to press the tone key when the tone was activated and the light key when the light was activated. Subjects were informed that on some trials both light and tone would be presented simultaneously. On these trials, the subject was instructed to press the key that was appropriate to the signal recognized first. RTs were measured in milliseconds.

Following 6 familiarization trials, subjects were given 20 simple RT trials, on which they were informed ahead of time which stimulus would be presented. Then, 20 choice RT trials were presented when the subject was not told ahead of time which stimulus would be activated. Interspersed with the 20 choice RT trials were 6 conflict trials, on which the tone and the light were presented simultaneously. There were 60 such conflict trials altogether (10 subjects, 6 trials each).

\section{RESULTS}

Mean simple RTs were $174 \mathrm{msec}$ and $195 \mathrm{msec}$ for tone and light trials, respectively. This difference was statistically significant at the .05 level. Mean RT values in the choice situation were $278 \mathrm{msec}$ and $283 \mathrm{msec}$ for tone and light trials, respectively.

Of the 60 conflict trials with simultaneous lighttone presentations, the light key was pressed 51 times. 
The tone key was pressed on the remaining nine trials. Following each conflict trial, subjects were asked if they were aware that both stimuli had been presented. Awareness was reported on 57 of 60 trials.

Mean RT for the 51 conflict trials, on which the light key was pressed, was $241 \mathrm{msec}$; mean RT on the 9 trials on which the tone key was pressed was $175 \mathrm{msec}$.

\section{DISCUSSION}

As in our earlier studies, simple tonal RT was significantly faster than simple light RT. Also, in line with our earlier reports, there were no significant RT differences between light trials and tone trials in the choice RT condition.

Once again, the visual dominance effect exhibited considerable robustness. Of 60 conflict trials, light keypresses occurred $85 \%$ of the time. The comparable figure from our earlier "freefield" data was $94 \%$. Thus, the use of earphones may have brought about a slight reduction in visual dominance, but the response tendency on conflict trials was still overwhelmingly in favor of the light.

Our hypothesis in conducting the present experiment was that manipulating the extent to which subjects must divide their attention between objects in space would influence the magnitude of the visual dominance effect. It is not clear that this aim was achieved, in that we still noted a high percentage of light-key responses on conflict trials. However, we have at least provided a further example of the variety of experimental conditions under which the visual dominance effect can be obtained.

The causal mechanism underlying visual dominance remains undiscovered. However, some of our earlier work (Colavita \& Weisberg, 1979), as well as studies by Egeth and Sager (1977) and Posner, Nissen, and Klein (1976), suggests that the visual dominance effect appears to be nonsensory (attentional) rather than sensory (some sort of masking).

\section{REFERENCES}

Colavita, F. B. Human sensory dominance. Perception \& Psychophysics, 1974, 16, 409-412.

Colavita, F. B., Tomko, R., \& Weisberg, D. Visual prepotency and eye orientation. Bulletin of the Psychonomic Society, 1976, 8, 581-582.

Colavita, F. B., \& Weisberg, D. A further investigation of visual dominance. Perception \& Psychophysics, 1979, 25, 345-347.

Egeth, H. E., \& Sager, L. C. On the locus of visual dominance. Perception \& Psychophysics, 1977, 22, 77-86.

Posner, M. I., Nissen, M. J., \& KLEIN, R. M. Visual dominance: An information-processing account of its origins and significance. Psychological Review, 1976, 83, 157-171.

(Received for publication February 9, 1982.) 